\title{
Pre-test ${ }^{68} \mathrm{Ga}$-PSMA-ligand PET/CT positivity in early biochemical recurrent prostate cancer after radical prostatectomy - validation of a prediction model
}

Pia Kraft ${ }^{1}$, Tobias Maurer ${ }^{2,4}$, Andrei Gafita' ${ }^{1}$, Markus Krönke ${ }^{1}$, Bernhard Haller ${ }^{3}$, Wolfgang A. Weber ${ }^{1}$, Matthias Eiber ${ }^{1}$ and Isabel Rauscher ${ }^{1 *}$

\begin{abstract}
Objectives: The aim of this study was the validation of a recently established comprehensive and compact prediction model for ${ }^{68} \mathrm{Ga}-\mathrm{PSMA}-11$-ligand positron-emission tomography (PET) positivity with an independent subsequent patient series.

Methods: A total of 292 consecutive patients with early biochemical recurrence after radical prostatectomy and PSA values between 0.2 and $1 \mathrm{ng} / \mathrm{ml}$ who underwent ${ }^{68} \mathrm{Ga}$-PSMA-11-ligand PET/computed tomography (CT) between January 2016 and June 2017 were retrospectively included. The cohort was divided into a very low PSA value $(0.2-0.5 \mathrm{ng} / \mathrm{ml}, n=151)$ and a low PSA value $(>0.5-1 \mathrm{ng} / \mathrm{ml}, n=141)$ subgroup. First, pre-test positivity probabilities for each patient were calculated according to the previously published comprehensive prediction model using all clinical variables (PSA value, ISUP grade group, T- and N-stage, patient under androgen deprivation therapy (ADT), previous radiation therapy) and the compact model using just the most predictive factors PSA value, ADT, and grade group. Then, all ${ }^{68} \mathrm{Ga}-\mathrm{PSMA}-11$-ligand PET/CTs were analysed by one experienced nuclear medicine physician, and the results were correlated to the calculated pre-test probabilities.

Results: In the very low PSA value subgroup, mean pre-test probability for positive findings in ${ }^{68} \mathrm{Ga}-\mathrm{PSMA}$-11-ligand PET/CT was 57\% (95\% Cl 55-60\%) according to the compact model and 59\% (95\% Cl 56-61\%) according to the comprehensive model. In the low PSA value subgroup, mean pre-test probability was $72 \%(95 \% \mathrm{Cl} 70-74 \%)$ in the compact model and $74 \%(95 \% \mathrm{Cl} 72-76 \%)$ in the comprehensive model. After image analysis, 59\% (89/151) of the patients in the very low PSA value subgroup revealed positive imaging findings. Seventy-nine percent (112/141) of the patients in the low PSA value subgroup presented with positive findings in the ${ }^{68} \mathrm{Ga}-\mathrm{PSMA}-11$-ligand PET/CT. The accuracy (AUC) of the prediction models was 0.71 (95\% Cl 0.65-0.78) for the compact model and 0.74 ( $95 \% \mathrm{Cl}$ 0.68-0.80) for the comprehensive model.

(Continued on next page)
\end{abstract}

\footnotetext{
* Correspondence: isabel.rauscher@tum.de

${ }^{1}$ School of Medicine, Klinikum rechts der Isar, Department of Nuclear

Medicine, Technical University of Munich, Ismaninger Str. 22, 81675 Munich,

Germany

Full list of author information is available at the end of the article
} 
(Continued from previous page)

Conclusion: External validation of the recently proposed prediction models showed a high concordance of the calculated pre-test probabilities and actual ${ }^{68} \mathrm{Ga}$-PSMA-11-ligand PET/CT findings in the validation cohort confirming the prediction models' ability to determine the presence of a positive lesion at ${ }^{68} \mathrm{Ga}$-PSMA-11-ligand PET. However, the predictive accuracy of the nomogram itself is suboptimal and should be used with caution. Furthermore, the model's generalizability may be hampered due to the study design (in-house validation). Nevertheless, given the limited health resources and the costs of hybrid imaging techniques, prediction models might be a benefit in patient selection.

Keywords: Biochemical recurrence, Prostate cancer, PSMA, External validation, Prediction model

\section{Introduction}

Prostate cancer (PC) is the second most common cancer in men worldwide [1]. After primary radical prostatectomy (RP), the recurrence rate ranges between 7 and $50 \%$ within 5 years after surgery $[2,3]$. In these cases, early detection and localization of recurrent disease is crucial for further disease management $[4,5]$. Conventional imaging such as computed tomography (CT) or magnetic resonance imaging as well as choline-based PET often fails to localize biochemical recurrence [4, $6-8]$. Since its first introduction in $2014,{ }^{68}$ Ga-PSMA11-ligand positron emission tomography/computed tomography (PET/CT) has been adopted rapidly around the world given its high sensitivity and specificity for the detection and localization PC lesions. The most accepted clinical indication for PSMA-ligand PET/CT is biochemically recurrent $\mathrm{PC}$ as various studies indicate superior detection efficacy in comparison to conventional imaging and choline-based PET, even at low PSA levels [9]. This is also reflected by the recent introduction into the EAU guidelines [4].

In uro-oncology, prediction nomograms are designed to help patients and their physicians to assess risk based on specific characteristics and predict the likely outcomes of treatment. In PC, the most established nomograms are the Partin tables or the Kattan nomograms predicting, for example, the extent of the cancer and long-term results following RP already prior surgery or the probability of remaining cancer recurrence-free after surgery $[10,11]$. Recently, both a compact and a full comprehensive version of a prediction model were introduced providing individualized predictions for positive ${ }^{68}$ Ga-PSMA-11-ligand PET/CT scan in patients with very early biochemical recurrence (PSA $0.2-1 \mathrm{ng} / \mathrm{ml}$ ). These can assist urologists in discussions with their patients on the use of ${ }^{68} \mathrm{Ga}$-PSMA-11-ligand PET/CT in specific clinical settings [12]. The compact model combines the three most predictive clinical variables (PSA level, androgen deprivation therapy (ADT), ISUP Grade Group (old Gleason score)) which is in line with several recent studies supporting the role of PSA, ADT, and Gleason score as independent predictive factors for
PSMA-ligand PET positivity [12-14]. The comprehensive model adds the variables primary $\mathrm{T}$ - and $\mathrm{N}$-stage, prior radiation therapy, and time interval from RP to PSMA-ligand PET/CT examination for calculation of PSMA-ligand PET positivity.

In order to further establish the previously proposed prediction models showing their applicability in different patient populations, we aimed to validate the prediction models estimating pre-test ${ }^{68}$ Ga-PSMA-11-ligand PET/ CT positivity in early biochemical recurrent PC patients after RP in a second, independent patient population.

\section{Material and methods \\ Patient population}

We extracted 2051 consecutive patients undergoing ${ }^{68}$ Ga-PSMA-ligand PET imaging from the institution's database (January 2016 to June 2017). Only patients who were examined by PET/CT and showed PSA values from 0.2 up to $1 \mathrm{ng} / \mathrm{ml}$ at time of ${ }^{68}$ Ga-PSMA-11-ligand PET after primary treatment with RP were included in this study. Details on patient selection are presented in Fig. 1. If several ${ }^{68}$ Ga-PSMA-11-ligand PET/CT

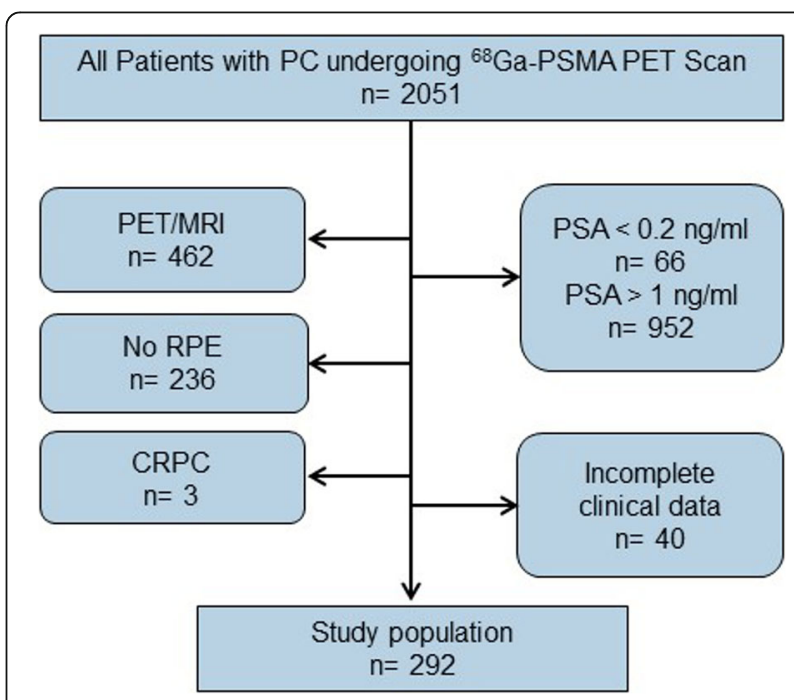

Fig. 1 Patient selection flow chart. PC, prostate cancer; RP, radical prostatectomy; CRPC, castration resistant prostate cancer 
examinations within one patient were available, only the first examination was included. All patients signed a written informed consent form for the purpose of anonymized evaluation and publication of their data. The study was approved by the Ethics Committee of the Technical University Munich (permit 5665/13).

\section{${ }^{68}$ Ga-PSMA-11-ligand PET/CT imaging}

Images were obtained with the ${ }^{68} \mathrm{Ga}$-labelled HBED-CC that was synthesized as described previously [15]. ${ }^{68} \mathrm{Ga}$ PSMA-11-ligand was applied to patients via an intravenous bolus (mean $1.76 \mathrm{MBq} / \mathrm{kg}$ body weight, IQR 1.18-1.94 MBq/kg; mean $131.5 \mathrm{MBq}$, IQR $102.3-160.6$ $\mathrm{MBq})$. PET acquisition was started at a mean time of $54.1 \pm 11.3 \mathrm{~min}$ after tracer injection (range 40-181 min; for two patients, compensatory increase in time per bed position due to late imaging acquisition). All patients underwent ${ }^{68} \mathrm{Ga}$-PSMA-11-ligand PET/CT on a Biograph mCT scanner (Siemens Medical Solutions, Erlangen, Germany). First, a diagnostic CT scan was performed in the portal venous phase $80 \mathrm{~s}$ after intravenous injection of contrast agent (Imeron 300) followed by the PET scan. All PET scans were acquired in 3D mode with an acquisition time of 3-4 min per bed position. Emission data were corrected for randoms, dead time, scatter, and attenuation and were reconstructed iteratively by an ordered-subsets expectation maximization algorithm (four iterations, eight subsets) followed by a postreconstruction smoothing Gaussian filter (5-mm full width at one-half maximum).

\section{Data analysis}

First, a pre-test positivity probability score (percentage with $95 \%$ confidence intervals) was calculated for each patient using the formula of the compact and the comprehensive prediction model described in Rauscher et al. [12]. In the second step, all ${ }^{68}$ Ga-PSMA-11-ligand PET/ CTs were evaluated by one experienced nuclear medicine physician (IR) blinded to the results of the prediction model and all clinical data of the patient $[16,17]$. All lesions suspicious for recurrent PC were noted and grouped into the following: (a) local recurrence, (b) lymph node metastases (pelvic/retroperitoneal vs. supradiaphragmatic location), (c) bone metastases, and (d) other visceral metastases (e.g. lung, liver, spleen).

\section{Statistical analysis}

All statistical analyses were performed with the SPSS software. For analysis, the patient cohort was divided into two subgroups: patients with very low PSA values $(0.2-0.5 \mathrm{ng} / \mathrm{ml})$ and patients with low PSA values (> $0.5-1 \mathrm{ng} / \mathrm{ml})$. Pre-test probabilities were calculated as previously described and correlated with the results from subsequent ${ }^{68} \mathrm{Ga}$-PSMA-11-ligand PET/CT analysis for all patients in each PSA subgroup [12]. Logistic regression models were fitted to the data to estimate odds ratios for $t$ variables. Receiver operating characteristics (ROC) analysis was conducted to compare associations of binary variables to ${ }^{68} \mathrm{Ga}$-PSMA-11-ligand PET positivity and the discriminatory ability of the prediction model. To validate the predicted and actual probabilities, calibration plots were calculated. All statistical tests were performed two-sided, and a significance level of $\alpha=5 \%$ was used.

\section{Results}

In total, 292 patients were included in this study. Patient characteristics are summarized in Table 1. One hundred fifty-one patients presented with very low PSA values $(0.2-0.5 \mathrm{ng} / \mathrm{ml})$ and 141 patients presented with low PSA values $(>0.5-1 \mathrm{ng} / \mathrm{ml})$, respectively.

\section{Calculated pre-test probabilities}

In the very low PSA value subgroup $(0.2-0.5 \mathrm{ng} / \mathrm{ml})$, mean pre-test probability for positive findings in ${ }^{68} \mathrm{Ga}$ PSMA-11-ligand PET/CT was calculated to be 57\% (95\% CI 55-60\%) according to the compact model and 59\% (95\% CI 56-61\%) according to the comprehensive model. For the low PSA value subgroup ( $>0.5-1 \mathrm{ng} / \mathrm{ml})$, the estimated mean pre-test probability was $72 \%(95 \%$ CI $70-74 \%)$ in the compact model and $74 \%$ (95\% CI $72-$ $76 \%)$ in the comprehensive model.

\section{Lesion detection and localization}

In total, ${ }^{68} \mathrm{Ga}$-PSMA-11-ligand PET/CT was positive in $68.8 \%(201 / 292)$ of all patients in the validation cohort, respectively. In the very low PSA value subgroup (0.2-

Table 1 Patients' clinical and pathologic characteristics

\begin{tabular}{|c|c|c|}
\hline Characteristics & $n=292$ & \\
\hline Age at PET/CT, median (years) & & $70($ IQR 65-74) \\
\hline \multirow[t]{2}{*}{ Primary Gleason score at RP } & $\leq 7$ & $204(69.9 \%)$ \\
\hline & $\geq 8$ & $88(30.1 \%)$ \\
\hline \multirow{2}{*}{$\begin{array}{l}\text { Pathologic primary tumour } \\
\text { stage }(\mathrm{pT})\end{array}$} & $\leq \mathrm{pT} 2 \mathrm{c}$ & $140(47.9 \%)$ \\
\hline & $\geq$ pT3a & $152(52.1 \%)$ \\
\hline \multirow{2}{*}{$\begin{array}{l}\text { Pathologic regional lymph } \\
\text { node stage (pN) }\end{array}$} & pNO & $230(78.8 \%)$ \\
\hline & $\mathrm{pN} 1$ & $62(21.2 \%)$ \\
\hline Additional treatment after $\mathrm{RP}^{1}$ & & $142(48.6 \%)$ \\
\hline Radiation therapy & & $107(36.6 \%)$ \\
\hline ADT (within the last 6 months) & & $35(12.0 \%)$ \\
\hline $\begin{array}{l}\text { PSA value prior to PET/CT, } \\
\text { median }(\mathrm{ng} / \mathrm{ml})\end{array}$ & & 0.50 (IQR 0.35-0.70) \\
\hline $0.2-0.5 \mathrm{ng} / \mathrm{ml}$ (very low) & $n=151$ & 0.35 (IQR 0.27-0.43) \\
\hline$>0.5-1.0 \mathrm{ng} / \mathrm{ml}$ (low) & $n=141$ & 0.71 (IQR 0.60-0.90) \\
\hline
\end{tabular}

$A D T$ androgen deprivation therapy, $R P$ radical prostatectomy

${ }^{1}$ Multiple secondary treatments within a patient possible 
$0.5 \mathrm{ng} / \mathrm{ml}), 59 \%(89 / 151)$ of the patients presented with positive imaging findings while $79 \%(112 / 141)$ of the patients in the low PSA value subgroup $(>0.5-1 \mathrm{ng} / \mathrm{ml}$ ) had a positive ${ }^{68}$ Ga-PSMA-11-ligand PET/CT scan (Table 2). The main site of lesions was pelvic/retroperitoneal lymph nodes, followed by bone and local recurrence. Detailed information on the localization of positive findings on ${ }^{68} \mathrm{Ga}$-PSMA-11-ligand PET according to PSA range is presented in Table 3. Representative examples are shown in Additional file 1: Figure S1.

\section{Accuracy of the prediction models}

In our study, the accuracy (area under the ROC curve (AUC)) of the prediction models was 0.71 (95\% confidence interval (CI) 0.65-0.78) for the compact model and 0.74 (95\% confidence interval $0.68-0.80$ ) for the comprehensive model (Fig. 2). This indicates a moderate to good discriminatory ability. Further, no significant differences were observed compared to the AUCs of our previously published prediction models ( $p$ values 0.376 and 0.242 in the compact and comprehensive model, respectively) [12].

Figure 3 displays the nomogram calibration plot as applied to the validation dataset. The predicted probability of the previously reported prediction model is represented on the $x$-axis, and the actual proportion of ${ }^{68}$ Ga-PSMA-11-ligand PET positivity is represented on the $y$-axis. In both the compact and comprehensive models, a tendency to slightly overestimate the actual positivity rate was observed for most of the patients. Only in patients with a very high or very low predicted positivity rate a slight underestimation of PET positivity was observed.

\section{Lesion validation}

Validation of positive ${ }^{68} \mathrm{Ga}$-PSMA-11-ligand PET/CT findings was available in $137 / 201$ patients (68 \%) with at least 1 of the following procedures: (a) targeted radiation therapy with consecutive PSA decline $\leq 0.2 \mathrm{ng} / \mathrm{ml}(n=$ 56), (b) positive histopathology after salvage lymph node dissection $(n=27)$, and (c) follow-up ${ }^{68}$ Ga-PSMA-11-ligand PET/CT confirming the initial suspicious lesion(s) or disappearance of suspected metastatic sites after local/systemic treatment and corresponding PSA decline $(n=17)$.

\section{Discussion}

Recently, two prediction models to estimate the a priori probability of ${ }^{68} \mathrm{Ga}$-PSMA-ligand PET/CT positivity in patients after RP presenting with biochemical recurrence (PSA level $0.2-1.0 \mathrm{ng} / \mathrm{ml}$ ) based on a variety of different clinical variables were established. The results of our validation study show a high concordance of the calculated pre-test probabilities and actual ${ }^{68}$ Ga-PSMA-11-ligand PET/CT findings in our recent patient cohort confirming the prediction models' ability to determine the probability of the presence of a positive lesion at ${ }^{68} \mathrm{Ga}$-PSMA-11-ligand PET (calculated probability: very low PSA 59\%/57\% and low PSA $74 \% / 72 \%$ for the comprehensive/compact model; suspicious lesions observed in ${ }^{68} \mathrm{Ga}$ PSMA-11-ligand PET 59\% and 79\% for very low and low PSA subgroup, respectively). The predicted pretest probabilities showed a slightly better congruence for the very low PSA subgroup; however, no statistical difference was observed. As there was no significant difference between the compact and comprehensive prediction model in this validation study, we recommend using the compact model for routine clinical use as several variables included in the original comprehensive model showed no statistical significance at multivariable analysis (e.g. $\mathrm{T}$ - and $\mathrm{N}$-stage, radiation therapy after surgery). The calculation of the compact model is easier and faster to apply as less clinical variables are necessary (only PSA level, grade group, and prescription of ADT).

The observed detection rate in our study is slightly higher than that reported in our previously published study $(69 \% \quad(201 / 292$ patients $)$ vs. $65 \% \quad(176 / 272$ patients) and in comparison to a study of AfsharOromieh et al. $(60 \%$; 137/227) in patients with a PSA value of $0.2-1 \mathrm{ng} / \mathrm{ml}[12,14]$. However, it has to be stated that the variance in detection rates is quite high in the literature and the numbers reported in our analysis are within these variations [18]. These variations might be due to the use of different types of PET/CT cameras, acquisition parameters, reconstruction algorithms, and/or the sensitivity/experience of the reader. In order to standardize image acquisition and evaluation and thus improve accuracy, precision, and repeatability of ${ }^{68} \mathrm{Ga}-\mathrm{PSMA}$ ligand $\mathrm{PET} / \mathrm{CT}$ examinations, several papers have been

Table 2 Subgroup analysis of pre-test probability and actual positive findings in ${ }^{68} \mathrm{Ga}$-PSMA-11-ligand PET

\begin{tabular}{llll}
\hline Patient subgroup & Compact model pre-test probability & Comprehensive model pre-test probability & Positive imaging findings \\
\hline Entire cohort & $67 \%(95 \%$ Cl 65-68\%) & $69 \%(95 \%$ Cl 66-71\%) & $69 \%(201 / 292)$ \\
Very low PSA $(0.2-0.5 \mathrm{ng} / \mathrm{ml})$ & $57 \%(95 \% \mathrm{Cl} 55-60 \%)$ & $59 \%(95 \% \mathrm{Cl} 56-61 \%)$ & $59 \%(89 / 151)$ \\
Low PSA $>0.5-1 \mathrm{ng} / \mathrm{ml})$ & $72 \%(95 \% \mathrm{Cl} 70-74 \%)$ & $74 \%(95 \% \mathrm{Cl} 72-76 \%)$ & $79 \%(112 / 141)$ \\
\hline
\end{tabular}


Table 3 Localization of positive findings on ${ }^{68} \mathrm{Ga}$-PSMA-11-ligand PET according to PSA range

\begin{tabular}{|c|c|c|c|c|c|}
\hline \multirow[t]{2}{*}{ PSA range } & \multicolumn{2}{|c|}{$0.2-0.5 \mathrm{ng} / \mathrm{ml}$ (very low) } & \multicolumn{2}{|c|}{$>0.5-1.0 \mathrm{ng} / \mathrm{ml}$ (low) } & \multirow[t]{2}{*}{$p$ value } \\
\hline & No. & $\%$ & No. & $\%$ & \\
\hline Total no. of patients with positive findings & $89 / 151$ & 58.9 & $112 / 141$ & 79.4 & $0.0003^{*}$ \\
\hline \multicolumn{6}{|c|}{ Localization of positive findings on ${ }^{68} \mathrm{Ga}-\mathrm{PSMA}-11$-ligand PET } \\
\hline Local & $24 / 151$ & 15.9 & $38 / 141$ & 27.0 & $0.0297^{*}$ \\
\hline LN pelvic/retroperitoneal & $58 / 151$ & 38.4 & $73 / 141$ & 51.8 & $0.0290^{*}$ \\
\hline LN supradiaphragmal & $7 / 151$ & 4.6 & $7 / 141$ & 5.0 & 0.9091 \\
\hline Bone & $30 / 151$ & 19.9 & $28 / 141$ & 19.9 & 0.8834 \\
\hline Visceral & $2 / 151$ & 1.3 & $4 / 141$ & 2.8 & 0.6215 \\
\hline
\end{tabular}

*Significant difference $p \leq 0.05$

published so far including the EANM/SNMMI procedure guidelines on ${ }^{68} \mathrm{Ga}$-PSMA-ligand PET/CT [16, 17, 19, 20]. Nevertheless, further efforts are needed for implementation in routine clinical practice as robust evidence is still lacking.

${ }^{68}$ Ga-PSMA-ligand PET/CT does not only detect but also localize the extent of recurrent disease. The main site of metastatic lesions in our study was pelvic/retroperitoneal lymph nodes, followed by bone and local recurrence which is in line with previous studies [12, 21]. Further, a substantial number of patients presented with supradiaphragmatic lymph node, bone, or visceral metastases, even at PSA values $0.2-0.5 \mathrm{ng} / \mathrm{ml}$ (see also Table 3). In patients with a PSA increase up to $0.5 \mathrm{ng} / \mathrm{ml}$, current guidelines recommend salvage radiotherapy. However, approximately $40 \%$ of them will not achieve an

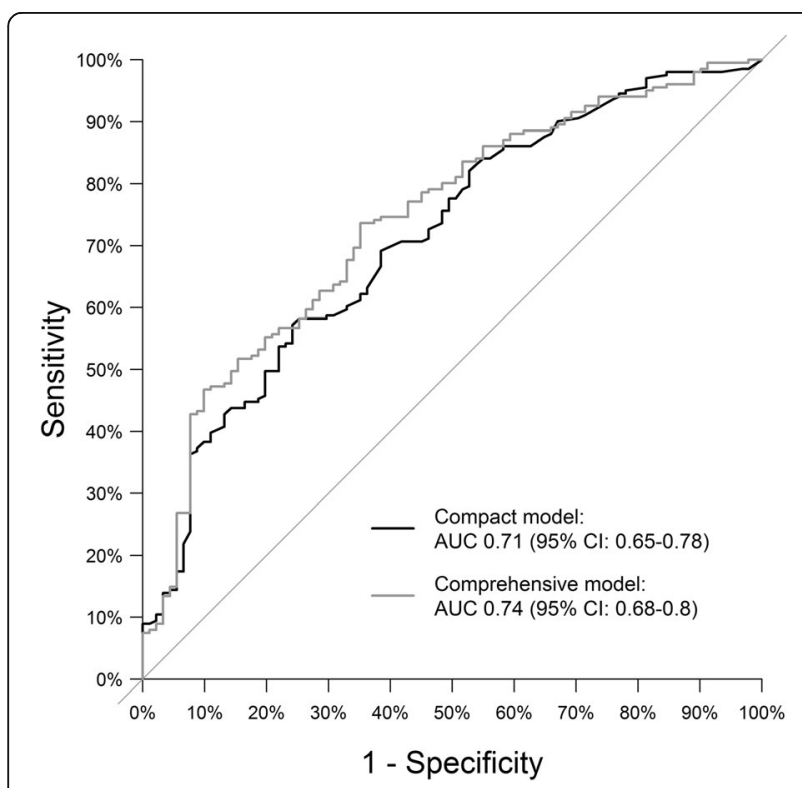

Fig. 2 ROC curves of the prediction models. The black line corresponds to the compact model, and the grey line to the comprehensive model (AUC, area under the curve; $\mathrm{Cl}$, confidence interval; $p$-values comparison to $A \cup C=0.5$ ) undetectable PSA value. Thus, ${ }^{68}$ Ga-PSMA-ligand PET/CT might not only modify potentially unsuccessful local salvage radiotherapy but also enable targeted surgical salvage procedures such as PSMAradioguided surgery $[22,23]$.

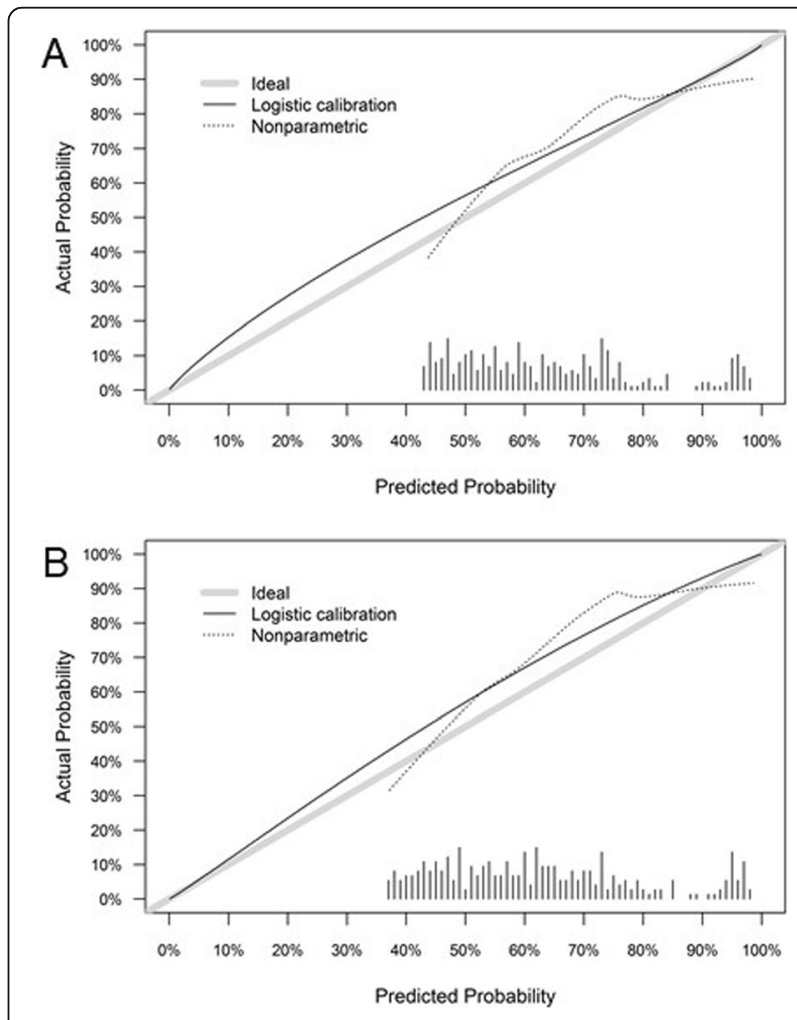

Fig. 3 Local regression nonparametric smoothing (calibration) plot of the compact (a) and comprehensive (b) prediction model showing the relationship between nomogram predictions and observed frequency of positive ${ }^{68} \mathrm{Ga}$-PSMA-11-ligand PET/CT examinations in the validation cohort. Perfect or ideal predictions correspond to the $45^{\circ}$ line (grey line). Points situated above the $45^{\circ}$ line correspond to overprediction in the validation cohort in comparison to the prediction model, whereas points estimated below the $45^{\circ}$ line correspond to underestimation in the validation cohort according to the prediction model (dotted black line). Vertical lines indicate the frequency distribution of predicted probabilities 
The prediction model itself is not able to specify probabilities below $40 \%$ and $30 \%$ in the compact and comprehensive model, respectively. Therefore, no justifiable cut-off can be defined in which clinical condition a ${ }^{68} \mathrm{Ga}$-PSMA-11-ligand PET examination is not required. Nevertheless, the prediction model is able to identify patients with recurrent disease in ${ }^{68} \mathrm{Ga}$ PSMA-11-ligand PET/CT with up to $98 \%$ certainty (highest score reachable in the prediction model based on clinical variables) who will potentially profit from lesion-targeted treatment. Therefore, the prediction models should rather be understood as a tool for a positive decision towards a PSMA-ligand PET/CT examination. As the results of the AUC were concordant in the patient cohort used for establishing the prediction nomogram and the one used for validation showing no significant difference, a further calibration of the prediction model based on our findings would not bring any added value and was not the aim of our study as well.

Nevertheless, we believe that it is of great interest to establish prediction nomograms being able to identify patients in which the examination will lead to a change in clinical management and might improve oncologic survivals. Thus, in patients at low risk of imaging-detected recurrence, PSMA-ligand PET/CT might not be recommended sparing expensive imaging that would not solve the clinical doubt of tumour recurrence and subsequent management would not have been changed as well. The huge interest in nomograms predicting PSMA-ligand PET positivity is also reflected by the recent introduction of a new prediction nomogram by Ceci et al. [24]. The main difference to the nomogram we validated is that Ceci et al. implemented PSA doubling time and included different clinical settings (first-time biochemical recurrence, biochemical recurrence after salvage therapy, biochemical persistence after radical prostatectomy, and advanced-stage PC before second-line systemic therapies). The predicted accuracy was $82 \%$ in their study and thus substantially higher than using the prediction nomograms of Rauscher et al. Further, a $40 \%$ probability threshold was identified to be the most accurate cut-off in counselling patients to $68 \mathrm{Ga}$ PSMA-11-PET/CT. However, multicentre external validation of their nomogram is still pending and thus, at present, cannot be suggested in routine.

There are several limitations to our study. First, we examined a subsequent patient cohort for external validation of the prediction model provided from the same institute using the same PET/CT scanner. To be precise, the patient group studied should have been from another site and using a different PET/CT scanner as variation of system performance is a known problem [25]. Therefore, the generalizability of the study may be hampered and multicentre validation in different sites is still mandatory. Nevertheless, we believe the nomogram is applicable on external patient populations as well as the study analyses a well-defined patient cohort meeting strict inclusion criteria. However, image acquisition parameters should be in line with the joint EANM/SNMMI guideline [19]. Further, in our study, ${ }^{68}$ Ga-PSMA-11 was used as PSMA-ligand although recently ${ }^{18} \mathrm{~F}$ PSMA-ligands are increasingly used because of various logistical advantages [13]. In future nomograms for ${ }^{18}$ F-PSMA-ligands, it can be anticipated that the low or even no excretion via the urinary tract increases detection probability in metastatic lesions located directly adjacent to the urinary bladder.

Recently, a higher number of lesions with increased PSMA-ligand uptake attributed to benign origin were observed in ${ }^{18} \mathrm{~F}$-labelled PSMA-ligand PET in comparison to ${ }^{68} \mathrm{Ga}$-PSMA-11 [26]. However, we do not think that this influences the correct establishment of a prediction nomogram as after adequate reader training, physicians should be well equipped to distinguish likely malignant lesions from those that are most probably benign. However, multivariable regression analysis with subsequent establishment of a prediction nomogram for ${ }^{18} \mathrm{~F}$-PSMA-ligand PET still has to show its practicability in the future. In addition, validation of PSMA-ligand positive imaging finding was not available in all patients; however, high specificity of ${ }^{68}$ Ga-PSMA-11-ligand PET has been proven in several studies by now $[6,21]$.

Above all, precise data proving that PSMA-guided therapy improves the disease-free and overall survival compared to conventional therapy is currently missing. Studies embracing this specific question are absolutely required in the future. At this point, prediction models would be a valuable tool to pre-clinically select patients in which a PSMA PET/CT would be beneficial.

\section{Conclusion}

Our study shows a high concordance of the calculated pre-test probabilities and ${ }^{68}$ Ga-PSMA-11-ligand PET/ CT findings confirming the prediction models' ability to determine the presence of a positive lesion at ${ }^{68}$ Ga-PSMA-11-ligand PET. However, the predictive accuracy of the nomogram itself is suboptimal and should be used with caution. In addition, due to inhouse validation, the model's generalizability may be reduced. Nevertheless, given the limited health resources and the costs of hybrid imaging techniques, prediction models might be a benefit in patient selection. 


\section{Supplementary information}

Supplementary information accompanies this paper at https://doi.org/10. 1186/s13550-020-0595-5

Additional file 1: Figure S1. Examples of ${ }^{68} \mathrm{Ga}-\mathrm{PSMA}-11$-ligand PET/CT examinations in patients with early recurrent PC after RP. Upper row shows CT datasets, lower row fused PSMA-ligand PET/CT studies. A, D: 58-year old male with biochemical recurrence (PSA $0.92 \mathrm{ng} / \mathrm{ml}$ ) 8 years after RP (pT2c, pN0, R0, GS 6) with intense, focal PSMA-ligand uptake in the central prostate fossa highly suggestive of local recurrence which was histopathologically proven (positive in HE staining and PSMA immunohistochemistry staining) after salvage surgery. B, E: 70-year old male with biochemical recurrence (PSA $0.21 \mathrm{ng} / \mathrm{ml}) 2$ years after RP (pT3a, pNo, RO, GS 7b) with intense PSMA-ligand uptake in a morphologically unobtrusive lymph node presacral highly suggestive of a single lymph node metastasis. Metastatic involvement of this lymph node was histologically proven (positive in HE staining and PSMA immunohistochemistry staining) after salvage lymphadenectomy. C, F: 67-year old male with biochemical recurrence (PSA $0.35 \mathrm{ng} / \mathrm{ml}) 5$ years after RP (pT3b, pN1, R0, GS 7) with intense accumulation of PSMA tracer in the right pelvic bone highly suggestive of bone metastasis. Follow-up PET/CT after salvage radiation therapy revealed no PSMA-ligand uptake anymore with corresponding PSA decline.

\section{Acknowledgements}

Not applicable

\section{Authors' contributions}

$I R, M E$, and WAW contributed to the design of the study. PK, TM, AG, MK, and $I R$ contributed to the collection of data. PK, TM, AG, MK, IR, and $B H$ contributed to the analysis/interpretation of data. PK and IR wrote the manuscript. PK, TM, AG, MK, IR, WAW, BH, and ME revised the manuscript. All authors read and approved the final manuscript.

\section{Funding}

There is no direct or indirect commercial financial incentive associated with publishing the article, and no extrainstitutional funding was achieved. The development of ${ }^{68} \mathrm{Ga}-\mathrm{PSMA}$ synthesis was supported by SFB 824 (DFG Sonderforschungsbereich 824, Project Z1) from the Deutsche Forschungsgemeinschaft, Bonn, Germany. ME received funding from the SFB 824 (DFG Sonderforschungsbereich 824, Project B11) from the Deutsche Forschungsgemeinschaft, Bonn, Germany. No other potential conflicts of interest relevant to this article exist.

\section{Availability of data and materials}

All data generated or analysed during this study are included in this published article (and its supplementary information files).

\section{Ethics approval and consent to participate}

All patients signed a written informed consent form for the purpose of anonymized evaluation and publication of their data. The study was approved by the Ethics Committee of the Technical University Munich (permit 5665/13).

\section{Consent for publication}

All patients signed a written informed consent form for the purpose of anonymized evaluation and publication of their data.

\section{Competing interests}

The authors declare that they have no competing interests.

\section{Author details}

${ }^{1}$ School of Medicine, Klinikum rechts der Isar, Department of Nuclear Medicine, Technical University of Munich, Ismaninger Str. 22, 81675 Munich, Germany. ${ }^{2}$ School of Medicine, Klinikum rechts der Isar, Department of Urology, Technical University of Munich, Ismaninger Str. 22, 81675 Munich, Germany. ${ }^{3}$ School of Medicine, Klinikum rechts der Isar, Institute of Medical Informatics, Statistics and Epidemiology, Technical University of Munich, Ismaninger Str. 22, 81675 Munich, Germany. ${ }^{4}$ Department of Urology,
Martini-Klinik Prostate Cancer Center, University Hospital

Hamburg-Eppendorf, Martini-Str. 52, 20246 Hamburg, Germany.

Received: 4 December 2019 Accepted: 16 January 2020

Published online: 03 February 2020

\section{References}

1. Bray F, et al. Global cancer statistics 2018: GLOBOCAN estimates of incidence and mortality worldwide for 36 cancers in 185 countries. CA Cancer J Clin. 2018;68(6):394-424.

2. Leyh-Bannurah, S.R., et al., Assessment of oncological outcomes after radical prostatectomy according to preoperative and postoperative cancer of the prostate risk assessment scores: results from a large, two-center experience. Eur Urol Focus, 2017

3. Pompe RS, et al. Oncologic and functional outcomes after radical prostatectomy for high or very high risk prostate cancer: European validation of the current NCCN(R) guideline. J Urol. 2017;198(2):354-61.

4. Mottet N., van den Bergh RCN, Briers E., Cornford P., De Santis M., Fanti S., Gillessen S., Grummet J., Henry A.M., Lam T.B., Mason M.D., Van der Kwast T. H., Van der Poel H.G., Rouvière O., Tilki D., Wiegel T., EAU - EANM - ESTRO ESUR - SIOG Guidelines on Prostate Cancer. 2019.

5. Bott SR. Management of recurrent disease after radical prostatectomy. Prostate Cancer Prostatic Dis. 2004;7(3):211-6.

6. Rauscher I, et al. Value of 68Ga-PSMA HBED-CC PET for the assessment of lymph node metastases in prostate cancer patients with biochemical recurrence: comparison with histopathology after salvage lymphadenectomy. J Nucl Med. 2016;57(11):1713-9.

7. Hovels AM, et al. The diagnostic accuracy of $C T$ and MRI in the staging of pelvic lymph nodes in patients with prostate cancer: a meta-analysis. Clin Radiol. 2008;63(4):387-95.

8. Schwenck J, et al. Intention to treat analysis of (68)Ga-PSMA and (11)Ccholine PET/CT versus CT for prostate cancer recurrences after surgery. J Nucl Med. 2019.

9. Perera $M$, et al. Gallium-68 prostate-specific membrane antigen positron emission tomography in advanced prostate cancer-updated diagnostic utility, sensitivity, specificity, and distribution of prostate-specific membrane antigen-avid lesions: a systematic review and meta-analysis. Eur Urol. 2019.

10. Kattan MW, et al. Evaluation of a nomogram used to predict the pathologic stage of clinically localized prostate carcinoma. Cancer. 1997;79(3):528-37.

11. Eifler JB, et al. An updated prostate cancer staging nomogram (Partin tables) based on cases from 2006 to 2011. BJU Int. 2013;111(1):22-9.

12. Rauscher l, et al. Efficacy, predictive factors, and prediction nomograms for (68)Ga-labeled prostate-specific membrane antigen-ligand positron-emission tomography/computed tomography in early biochemical recurrent prostate cancer after radical prostatectomy. Eur Urol. 2018;73(5):656-61.

13. Giesel FL, et al. Detection efficacy of (18)F-PSMA-1007 PET/CT in 251 patients with biochemical recurrence of prostate cancer after radical prostatectomy. J Nucl Med. 2019:60(3):362-8.

14. Afshar-Oromieh A, et al. Diagnostic performance of (68)Ga-PSMA-11 (HBED-CC) PET/CT in patients with recurrent prostate cancer: evaluation in 1007 patients. Eur J Nucl Med Mol Imaging. 2017;44(8):1258-68.

15. Martin $\mathrm{R}$, et al. Cationic eluate pretreatment for automated synthesis of [(6)(8)Ga]CPCR4.2. Nucl Med Biol. 2014;41(1):84-9.

16. Eiber $\mathrm{M}$, et al. Prostate cancer molecular imaging standardized evaluation (PROMISE): proposed miTNM classification for the interpretation of PSMAligand PET/CT. J Nucl Med. 2018;59(3):469-78.

17. Rauscher I, et al. (68)Ga-PSMA ligand PET/CT in patients with prostate cancer: how we review and report. Cancer Imaging. 2016;16(1):14

18. Perera $M$, et al. Sensitivity, specificity, and predictors of positive (68)Gaprostate-specific membrane antigen positron emission tomography in advanced prostate cancer: a systematic review and meta-analysis. Eur Urol. 2016;70(6):926-37.

19. Fendler WP, et al. (68)Ga-PSMA PET/CT: Joint EANM and SNMMI procedure guideline for prostate cancer imaging: version 1.0. Eur J Nucl Med Mol Imaging. 2017;44(6):1014-24.

20. Fanti $\mathrm{S}$, et al. Development of standardized image interpretation for ${ }^{68} \mathrm{Ga}$-PSMA PET/CT to detect prostate cancer recurrent lesions. Eur J Nucl Med Mol Imaging. 2017:44(10):1622-35

21. Eiber $M$, et al. Evaluation of hybrid (6)(8)Ga-PSMA ligand PET/CT in 248 patients with biochemical recurrence after radical prostatectomy. J Nucl Med. 2015;56(5):668-74. 
22. Horn T, et al. Single lesion on prostate-specific membrane antigen-ligand positron emission tomography and low prostate-specific antigen are prognostic factors for a favorable biochemical response to prostate-specific membrane antigen-targeted radioguided surgery in recurrent prostate cancer. Eur Urol. 2019;76(4):517-23.

23. Maurer T, et al. (99m)Technetium-based prostate-specific membrane antigen-radioguided surgery in recurrent prostate cancer. Eur Urol. 2019; 75(4):659-66.

24. Ceci F, et al. Prediction nomogram for (68)Ga-PSMA-11 PET/CT in different clinical settings of PSA failure after radical treatment for prostate cancer. Eur J Nucl Med Mol Imaging. 2020:47(1):136-46.

25. Rausch I, et al. Variation of system performance, quality control standards and adherence to international FDG-PET/CT imaging guidelines. A national survey of PET/CT operations in Austria. Nuklearmedizin. 2014;53(6):242-8.

26. Rauscher I, et al. Matched-pair comparison of (68)Ga-PSMA-11 PET/CT and (18)F-PSMA-1007 PET/CT: frequency of pitfalls and detection efficacy in biochemical recurrence after radical prostatectomy. J Nucl Med. 2020;61(1):51-7.

\section{Publisher's Note}

Springer Nature remains neutral with regard to jurisdictional claims in published maps and institutional affiliations.

\section{Submit your manuscript to a SpringerOpen ${ }^{\circ}$ journal and benefit from:}

- Convenient online submission

- Rigorous peer review

- Open access: articles freely available online

- High visibility within the field

- Retaining the copyright to your article

Submit your next manuscript at $\boldsymbol{\triangleright}$ springeropen.com 